\title{
Processo Integrado de Gerenciamento Quantitativo para Melhoria de Processos
}

\author{
Leonardo Romeu, Jorge Luis Nicolas Audy \\ Faculdade de Informática (FACIN) \\ Pontifícia Universidade Católica do Rio Grande do Sul (PUCRS) \\ 90.619-900 - Porto Alegre - RS - Brasil \\ leonardo.romeu@pucrs.br, audy@pucrs.br
}

\begin{abstract}
During the last years there have been many discussions about poor quality and low productivity in worldwide software industry which reflects on customer dissatisfaction and financial losses of huge proportions. In this context, defining methodologies to discipline the software development process is a critical factor of success. This paper aims to present a quantitative management process for process improvement. It is based on integration among BSC, CMMI and Six Sigma and is specified on EPF Composer. Besides that, a case study has been performed to evaluate the usage of the process and its results are discussed in this paper.
\end{abstract}

Resumo. Atualmente muito é discutido acerca da baixa qualidade $e$ produtividade da indústria mundial de software, refletindo-se na insatisfação de seus usuários e prejuízos financeiros de enormes proporções. Neste contexto, a definição de metodologias para disciplinar o processo de desenvolvimento de software é um fator crítico de sucesso para as organizações. Este artigo tem o objetivo de apresentar um processo de gerenciamento quantitativo para melhoria de processos. Este processo é baseado na integração entre BSC, CMMI e Six Sigma e foi especificado utilizando a ferramenta EPF Composer. Além disso, foi realizado um estudo de caso para avaliar a utilização do processo e os resultados são discutidos neste artigo.

\section{Introdução}

A Qualidade é, hoje, o grande motivador em todas as áreas de atividade humana e não poderia ser diferente em sistemas de informação, mais especificamente no desenvolvimento de software. Atualmente, muito é discutido acerca da baixa qualidade e produtividade da indústria mundial de software, refletindo-se na insatisfação dos seus usuários e em prejuízos financeiros de enormes proporções. (Oliveira, 2005)

As organizações desenvolvedoras de software têm almejado a excelência na qualidade de seus produtos em decorrência da crescente exigência de clientes. Elas também têm buscado minimizar custos, aumentar produtividade e cumprir prazos, a fim de se manterem competitivas no mercado (Donegan, 2005).

Um dos esforços mais significativos, na área de qualidade, corresponde à definição de metodologias voltadas a disciplinar o processo de desenvolvimento através do estabelecimento de etapas bem definidas, proporcionando, desta forma, um 
mecanismo de controle para o processo (Donegan, 2005). Desta forma, vários modelos, metodologias e métodos são oferecidos às organizações visando proporcionar melhoria de processos e conseqüente satisfação dos clientes. Os esforços de melhoria de processos devem estar alinhados para convergirem na mesma direção. Segundo Covatti (Covatti , 2007), o uso de um método integrado de implementação de melhoria oferece às organizações a oportunidade de concentrar todos os seus esforços em direção a um objetivo comum, a melhoria contínua de seus processos e produtos.

De acordo com Vasques (Vasques, 2006) a integração entre BSC, CMMI e Six Sigma pode prover alinhamento estratégico a programas de melhoria de processo. O alinhamento estratégico de programas de melhoria é fator crítico de sucesso para obtenção de patamares elevados de maturidade. Para Siviy (Siviy, 2005) e Pickerill (Pickerill, 2005), a utilização do Six Sigma como metodologia de implantação de processos em conjunto com o CMMI se constitui numa forma de acelerar o desenvolvimento e implantação de processos em organizações. Além disso, consideram o CMMI e o Six Sigma como complementares, à medida que o primeiro fornece um guia de boas práticas para processos enquanto o outro a forma de implementação dessas boas práticas.

Neste contexto, esta pesquisa apresenta um processo de gerenciamento quantitativo para melhoria de processos, em extensão à proposta apresentada por Covatti (Covatti, 2007): Método de Integração entre BSC, CMMI e Six Sigma (MIBCIS). O gerenciamento quantitativo é utilizado como um agente propulsor de melhoria contínua, ou seja, o estabelecimento de um sistema de medição capaz de prover subsídios para guiar as ações de melhoria de processo de forma alinhada à estratégia de negócio.

Em seguida, é abordado o referencial teórico que fundamentou a elaboração do processo proposto. Mais adiante, é apresentada uma visão geral do processo abordando as principais atividades e produtos de cada subprocesso. Além disso, são apresentados dados empíricos obtidos de um estudo de caso realizado em uma organização de software. Por fim, são apresentadas as considerações finais.

\section{Referencial Teórico}

\subsection{Balanced Scorecard}

Kaplan e Norton (Kaplan, 1997) definiram inicialmente o Balanced Scorecard (BSC) como um sistema de mensuração do desempenho e, posteriormente, como um sistema de gestão estratégica.

O BSC fornece às organizações uma visão compreensível e abrangente do negócio, focalizando os esforços de gestão, controle e melhoria, ao definir um grupo de medidas que controlem as atividades mais críticas dentro da organização. O nome Balanced Scorecard reflete o equilíbrio entre os objetivos de curto e longo prazo; entre medidas financeiras e não-financeiras; entre indicadores de tendência e ocorrências; entre perspectiva interna e externa do desempenho. É organizado entorno de quatro perspectivas: financeira, do cliente, interna e de inovação e aprendizado. Desta forma, o BSC é um sistema de gestão estratégica que traduz a missão e a estratégia da organização em objetivos e medidas através de um conjunto coerente de indicadores- 
chave de desempenho (KPI - Key Performance Indicators) de acordo com a visão e as expectativas da empresa a longo prazo. (Kaplan, 1997)

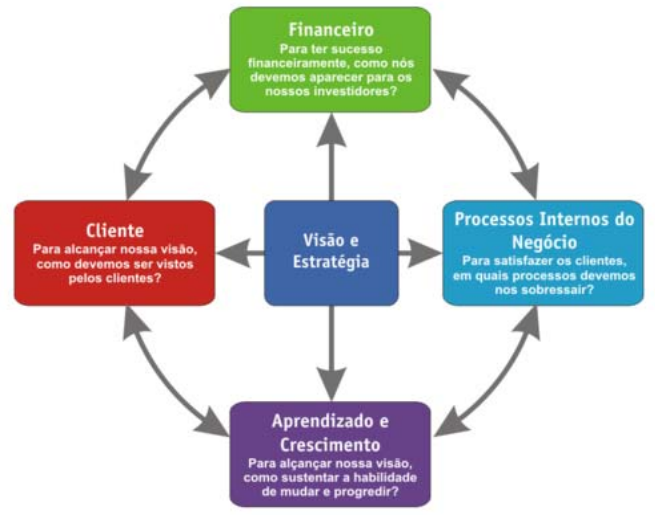

Figura 1 - Perspectivas BSC

Assim, o BSC proporciona ajustes e a mudança da própria estratégia, durante o processo de implementação, de forma que não só mensura como também estimula mudança. Na Figura 1, podemos observar as quatro perspectivas do BSC.

\subsection{CMMI}

O Capability Maturity Model Integration (CMMI) desenvolvido pelo Software Engineering Institute (SEI) é uma evolução do Capability Maturity Model (CMM) e procura estabelecer um modelo único para processos coorporativos, integrando diferentes modelos e disciplinas.

De uma forma resumida, o CMMI pode ser considerado um guia de boas práticas, as quais influenciarão a maneira pela qual uma organização desenvolve seus produtos e serviços. Desta forma, pode-se entender o CMMI como um conjunto de requisitos para processos, ou seja, o que um processo de trabalho de classe mundial deveria contemplar. O CMMI dá um conjunto robusto de orientações que, se bem interpretadas e adaptadas, respeitando-se o contexto de cada empresa, levam a melhorar a qualidade, produtividade e eficácia das organizações que os aplicam.

Existem duas formas de implementação do modelo CMMI, elas provêem às organizações a opção de implantá-lo apenas em um processo ou área da organização ou na organização como um todo. Essas formas são conhecidas como "Contínua" e "Por Estágio”.

A representação “Contínua” é uma abordagem mais flexível para o processo de melhoria e é definida em termos de níveis de potencialidade de processo. Nesta representação, a organização pode escolher uma área singular de processo ou trabalhar diversas áreas que estejam fortemente alinhadas com os objetivos de negócio da organização.

Já a representação por “Estágios” provê às organizações uma forma sistemática para melhoria de processos, pois como o nome já diz, a melhoria acontece em estágios, chamados de níveis de maturidade, sendo que cada nível atingido serve como base para o próximo (Ahern, 2003). 
Na Figura 2, podemos observar os cinco níveis de maturidade da representação por estágios do CMMI.

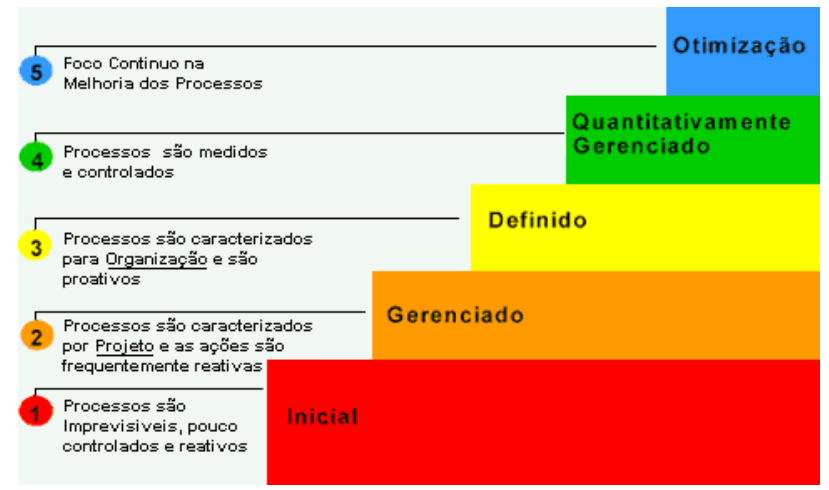

Figura 2 - Níveis de Maturidade

O CMMI é composto por Áreas de Processo (PA) que são um agrupamento de práticas relacionadas a uma área que, quando executadas coletivamente, satisfazem um grupo de metas consideradas importantes para significativas melhorias na área em questão. As áreas de processo são divididas em quatro categorias: Gerenciamento de Processos, Gerenciamento de Projetos, Engenharia e Suporte. Cada PA também está associada a um nível de maturidade na representação por “Estágios” do modelo (Ahern, 2003).

\subsection{Six Sigma}

Six Sigma é uma metodologia de melhoria de processo criada pela Motorola que utiliza a análise estatística para identificar causas primárias de problemas. Para medição de processo utiliza, por exemplo, a métrica de 3,4 defeitos por milhão de oportunidades (Fehlmann , 2002).

O objetivo fundamental do Six Sigma é implementar uma estratégia baseada em medições, que foca na melhoria de processo e redução de variação através do uso de um de seus modelos: DMAIC ou DMADV (Hayes, 2003).

A metodologia Six Sigma faz uso de ferramentas e métodos estatísticos para definir, medir, analisar, incorporar e controlar os processos ou produtos existentes, com a finalidade de alcançar etapas ótimas e que gerará um ciclo de melhoria contínua, conforme mostra a Figura 3.

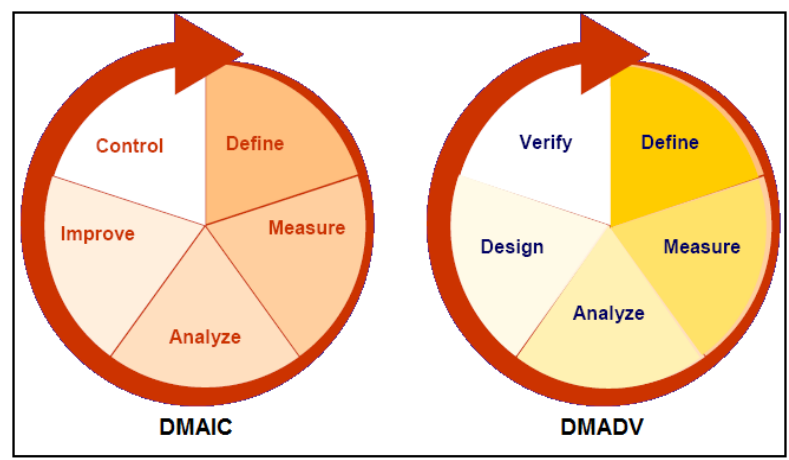

Figura 3 - Ciclos DMAIC e DMADV 
O modelo DMAIC é um sistema de melhoria para processos que necessitam de melhorias incrementais, sendo este o mais adotado pelas organizações para produtos e projetos já existentes. Ele corresponde a um acrônimo para as suas cinco fases: definir (Define), medir (Measure), analisar (Analyse), melhorar (Improve) e controlar (Control) (Eckes, 2001).

O modelo DMADV é utilizado para novos projetos/produtos. É uma adaptação do método DMAIC. É utilizado por uma metodologia denominada Design for Six Sigma (DFSS) que foi desenvolvida para fornecer aos seus usuários a habilidade de prever e prevenir defeitos na fase de desenvolvimento de um produto, serviço ou processo, ou seja, durante a fase de construção (Hayes, 2003).

\subsection{Trabalhos Relacionados}

Um dos principais estudos relacionados para esta pesquisa é o Método de Integração entre BSC, CMMI e Six Sigma (MIBCIS) proposto por Covatti (Covatti, 2007).

O objetivo principal do MIBCIS é prover uma estrutura contínua para a melhoria da qualidade de forma alinhada aos objetivos estratégicos da organização. Isto é obtido através do uso do Balanced Scorecard (BSC), que é uma ferramenta para medir e transmitir os objetivos estratégicos de uma organização, do CMMI, fonte das melhores práticas em desenvolvimento de software para instanciarmos framework de processo específico, e da metodologia Six Sigma para fornecer um mecanismo de melhoria continua de produtos e processos.

O método é dividido basicamente em três níveis: (1) Organizacional/Estratégico, (2) Projetos e Sistemas e (3) Melhoria Contínua. Na Figura 4, podemos ver a estrutura básica.

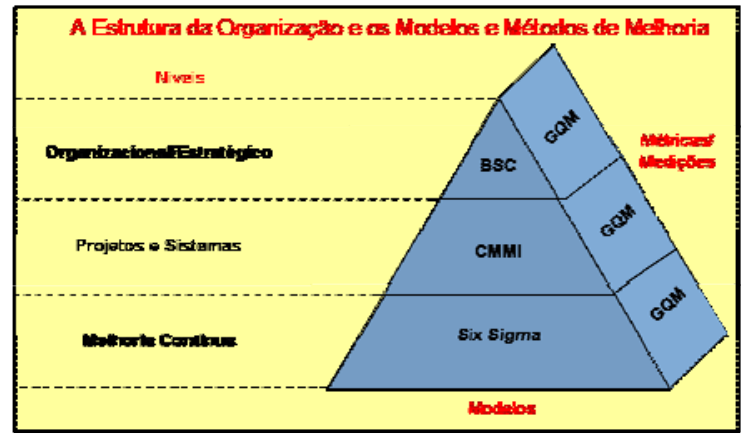

Figura 4. MIBCIS nos três níveis organizacionais

De forma bastante sucinta, uma das maiores contribuições desta evolução é a especificação da integração proposta através de um processo. Isto significa que existirá uma melhor descrição das atividades em todos os níveis do modelo, definindo claramente os atores que as executam bem como suas entradas e saídas esperadas. Outra contribuição é o foco no gerenciamento quantitativo como núcleo desta integração. $O$ desenvolvimento de uma ferramenta de apoio para automatizar o processo de obtenção e análise de indicadores e métricas também faz parte do escopo deste trabalho.

Segundo Vasques (Vasques, 2006), a composição entre as melhores práticas do CMMI, a metodologia Six Sigma e as diretrizes do BSC oferece o melhor em termos de objetividade e benefícios tangíveis na busca pela excelência e maturidade no desenvolvimento de aplicações de TI. 


\section{Processo Proposto}

O processo proposto é composto por quatro subprocessos, os quais possuem um conjunto de atividades correlatas que são executadas de forma assíncrona. Analogamente ao modelo de referência, MIBCIS, os processos estão organizados da seguinte forma, como mostra a Figura 5.

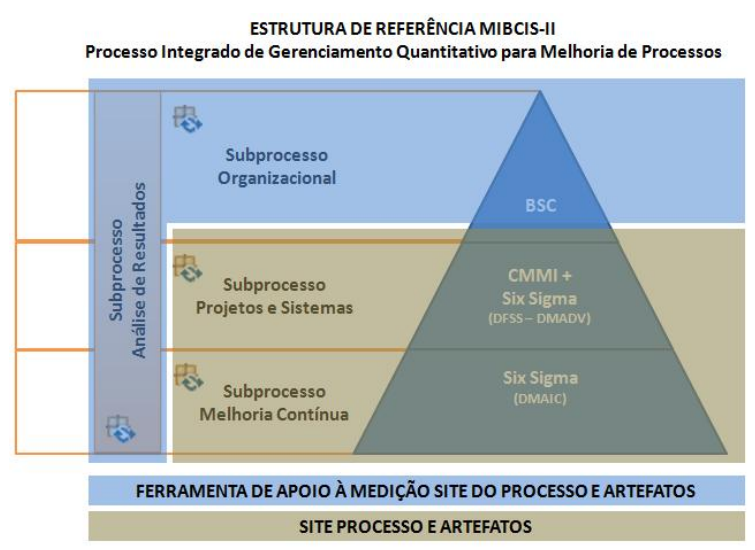

Figura 5 - Visão Geral Processo

O processo foi especificado utilizando a ferramenta Eclipse Process Framework Composer (EPF Composer). Nesta ferramenta, foram especificados os atores, tarefas e atividades conforme a estrutura da mesma, seguindo o padrão SPEM 2.0. Esta ferramenta possibilitou a criação de uma publicação do processo para posterior utilização em estudo de caso. Além disso, foi especificada e desenvolvida uma ferramenta de apoio à medição para suporte do gerenciamento quantitativo proposto no processo. Maiores detalhes da implementação e utilização de tal ferramenta serão discutidos em relato de experiência.

O subprocesso Organizacional, Figura 6, deve ser executado cada vez que a organização necessitar rever seu planejamento estratégico e seus objetivos de negócio. O planejamento estratégico, bem como suas revisões, é realizado pontualmente e espera resultados a longo prazo. Portanto o subprocesso organizacional será executado somente nestas ocasiões ou, no mínimo, uma primeira vez quando da adoção do processo proposto. Através da execução deste subprocesso, o planejamento e os objetivos estratégicos são transformados em mapas estratégicos e indicadores de desempenho. Estes indicadores de desempenho compõem a base para a gestão quantitativa de uma organização.

A atividade Balanced Scorecard compreende, então, o diagrama da Figura 7. As principais entradas são: o planejamento estratégico, objetivos de medição e necessidades de informação. Os produtos deste processo são os mapas estratégicos e os indicadores de desempenho definidos. 


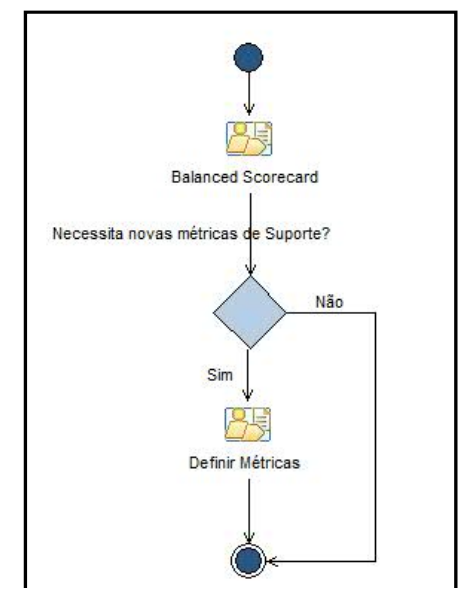

Figura 6 - Subprocesso Organizacional

A atividade Definir Métricas aparece em outros subprocessos e será mostrada somente nesta seção. A Figura 8, mostra as tarefas que devem ser realizadas para definir uma métrica. As principais entradas são necessidades de informação, framework de processos ou indicador de desempenho. O produto de saída desta atividade é a atualização das métricas da organização configuradas diretamente na ferramenta de apoio. Tal atividade só é necessária quando um indicador for composto por informações que ainda não estão suportadas pelo processo de medição da organização.

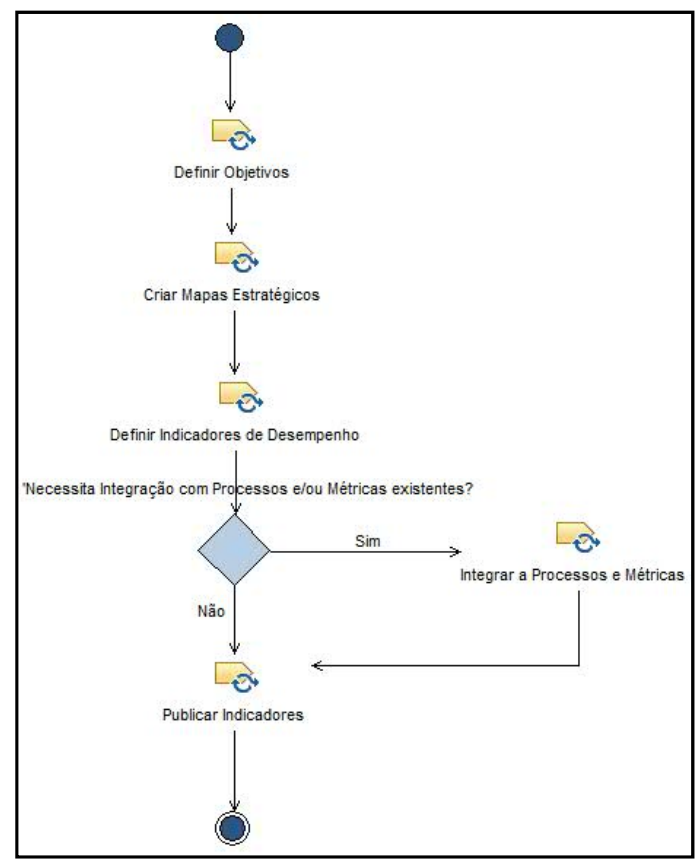

Figura 7. Balanced Scorecard

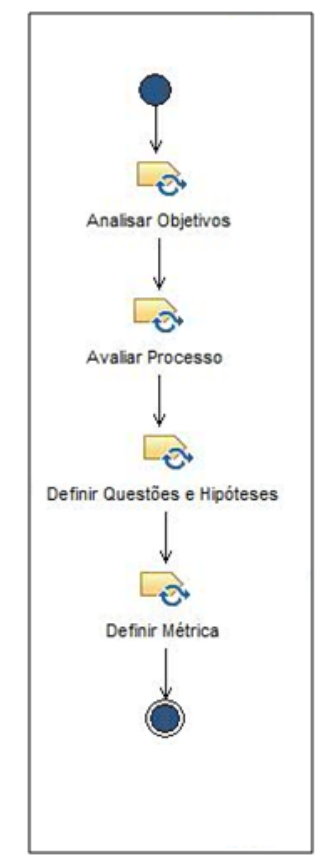

Figura 8. Definir Métricas

O subprocesso de Projetos e Sistemas, Figura 9, tem por objetivo definir uma seqüência de atividades para a criação de um framework de processos para a organização de forma alinhada aos objetivos estratégicos de negócio e em conformidade 
com as melhores práticas do CMMI. Deve ser executado cada vez que uma alteração estratégica tiver impactos em processos ou quando da adoção do processo proposto para atingir um nível de maturidade ou capacidade do modelo CMMI.

A principal atividade desse subprocesso é Desenvolver Processos (DMADV), baseada na metodologia conhecida como Design for Six Sigma (DFSS), detalhada na Figura 10. Além de desenvolver um novo processo, é necessário avaliar o impacto deste nas métricas existentes na organização através da tarefa Avaliar Impacto em Métricas, bem como definir novas métricas para suportá-lo. São entradas para este processo os objetivos de negócio e o modelo CMMI, como guia de referência para certo nível de maturidade definido pelo negócio. Os produtos de saída são processos, com uma determinada aderência às áreas de processo do modelo CMMI, e métricas para este novo processo.

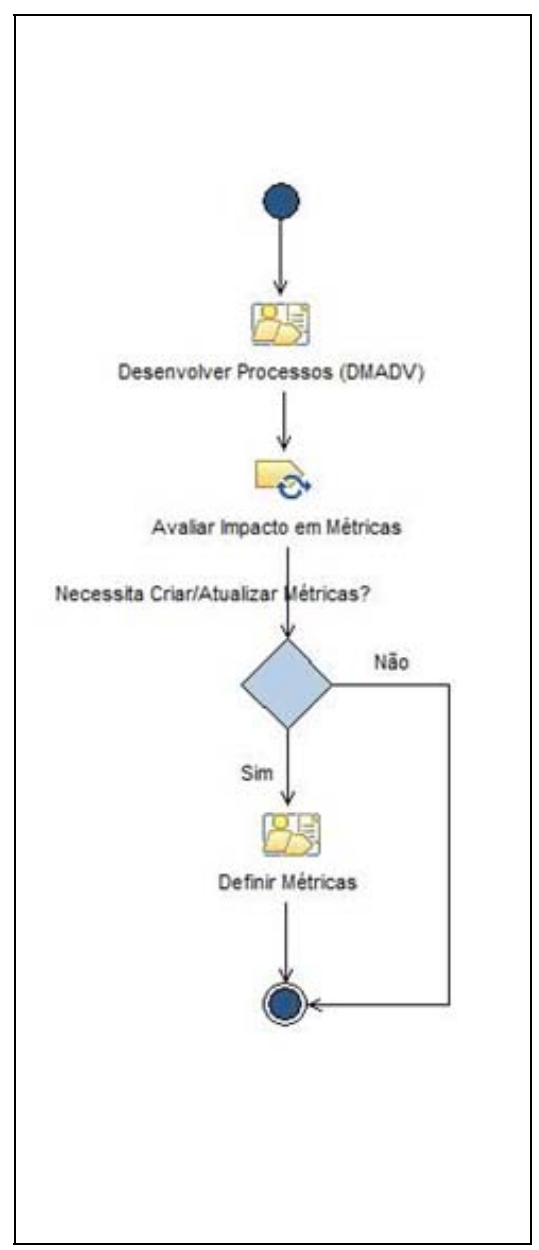

Figura 9. Subprocesso Projetos e Sistemas

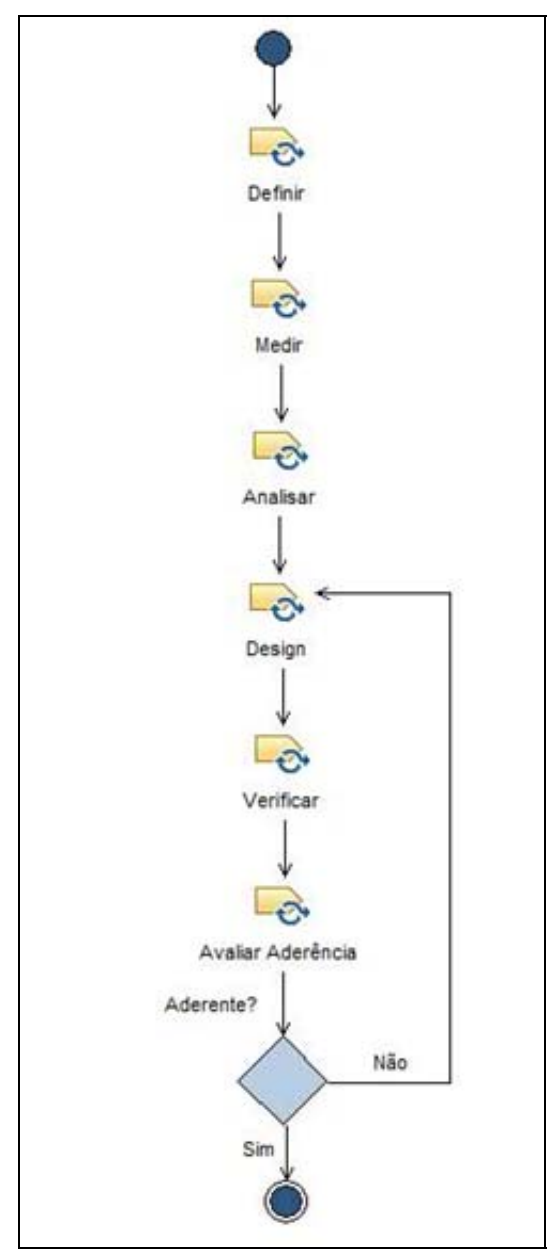

Figura 10. Desenvolver Processos (DMADV)

O subprocesso de Melhoria Contínua, Figura 11, visa transformar oportunidades de melhoria em atualizações de processos para garantir um melhor desempenho à organização. Essas oportunidades de melhoria podem vir de sugestões dos 
colaboradores ou através de alguma ação decorrente do processo de análise de resultados. Este subprocesso, como o nome já diz, deve ser executado continuamente para todas as melhorias identificadas de forma a avaliar e implementar cada melhoria utilizando um projeto DMAIC ou rejeitar e prover o feedback necessário.

Os projetos de melhoria em processos são realizados utilizando o fluxo de atividades da Figura 12 que é baseado no ciclo de melhoria da metodologia Six Sigma.

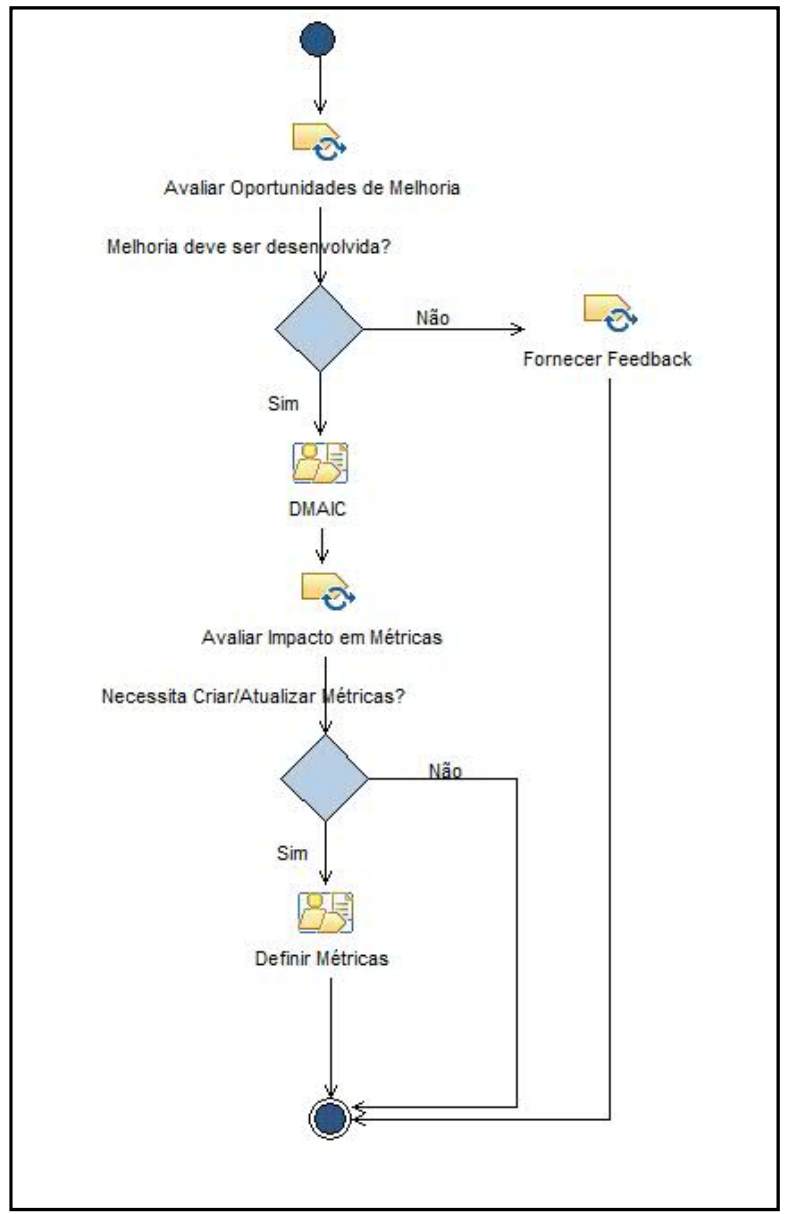

Figura 11. Processo Melhoria Contínua

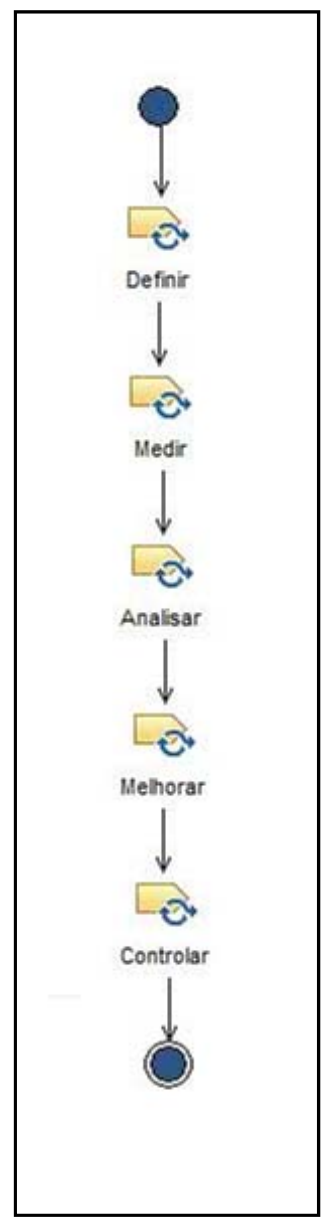

Figura 12. Fluxo DMAIC

Assim como no subprocesso Projetos e Sistemas, após uma melhoria de processo deve ser avaliado o impacto em métricas, através da tarefa Avaliar Impacto em Métricas, e/ou executada a atividade Definir Métricas, já descrita anteriormente.

O subprocesso de Análise de Resultados deve ser conduzido conforme a periodicidade de análise definida para os indicadores e métricas da organização. Este processo de análise deve ser executado em diferentes níveis organizacionais, a começar pela direção para indicadores de desempenho organizacionais até gestores de projetos para métricas específicas dos projetos. 


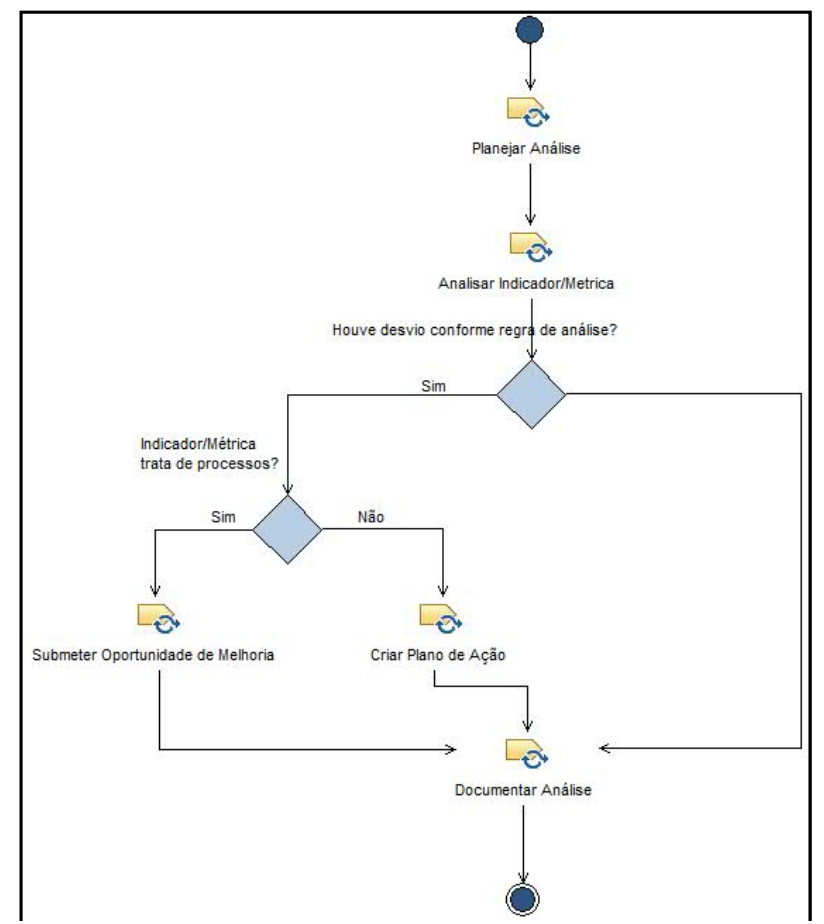

Figura 13. Processo de Análise de Resultados

Como decorrência deste subprocesso, é esperada a definição de ações de tratamento de desvios, bem como identificação de oportunidades de melhoria de processo, como pode ser visto na Figura 13. Estas oportunidades de melhoria de processo servem como entrada para o subprocesso de melhoria contínua. A documentação da análise de resultados também é uma saída deste processo e serve como evidência da ocorrência e institucionalização do mesmo.

\section{Estudo de Caso}

Um estudo de caso foi conduzido para avaliar a aplicabilidade do processo em uma organização de software. Tal estudo envolveu a execução do processo através da criação das instâncias do mesmo. O processo, as instâncias e a ferramenta de apoio foram apresentados a um conjunto de colaboradores da organização que responderam a um questionário construído para avaliar a aplicabilidade do processo. O questionário é composto por questões em escala Likert de 5 níveis, variando de "Discordo Totalmente" (com peso 1) a "Concordo Totalmente” (com peso 5). O questionário foi dividido em dimensões específicas para avaliar cada subprocesso.

\subsection{Caracterização da Organização}

A unidade onde o estudo foi aplicado está localizada na cidade de Porto Alegre (RS). Possui cerca de 200 colaboradores entre funcionários diretos, estagiários e terceiros, atuando em projetos que atendem aos seus clientes em diferentes linhas tecnológicas. A organização está estruturada internamente em centros de competência que atendem as necessidades de projetos nas diferentes linhas tecnológicas. 
Foi reconhecida como uma organização CMMI nível 2 em Março de 2005, após 10 meses de trabalho e, atualmente, encontra-se com o projeto de certificação nível 3 do CMMI DEV v1.2 em andamento.

\subsection{Análise dos Resultados}

O questionário foi dividido em três perfis de respondentes para melhor avaliar cada subprocesso. Responderam ao questionário diretores, gerentes de áreas e analistas de processo membros do Grupo de Engenharia de Processos (EPG) da organização totalizando 11 respondentes.

A primeira dimensão do questionário, relativa aos dados demográficos, teve como objetivo identificar os respondentes quanto à formação acadêmica e profissional. A consolidação dos principais itens desta dimensão do questionário se encontra na Tabela 1.

Tabela 1. Resultados Questionário - Dimensão 1

\begin{tabular}{|c|}
\hline Ítem Resultado \\
\hline Idade Média de idade 35 anos. Idade mínima 26 e máxima 46. \\
\hline Escolaridade Maioria com pós graduação ou mestrado. Dois respondentes com graduação incompleta e 1 com doutorado. \\
\hline Curso Divisão homogênea entre as áreas de Administração de Empresas e Sistemas de Informação \\
\hline Experiência Média de experiência 14 anos, tendo como mínimo 4 anos e máximo 22 anos. \\
\hline
\end{tabular}

Cabe ressaltar como resultado desta dimensão do questionário, que a experiência profissional dos respondentes é longa, como pode ser observado através da média de 14 anos. Em conjunto com a experiência, o nível de formação acadêmica também é elevado, fato que agrega relevância aos demais resultados do questionário, mesmo que o número de respondentes seja reduzido.

A segunda dimensão do questionário visava identificar a percepção dos respondentes quanto à aplicação do Subprocesso Organizacional. A consolidação dos resultados é apresentada na Tabela 2.

Tabela 2. Resultados Questionário - Dimensão 2

\begin{tabular}{|c|c|c|c|c|c|c|c|}
\hline Questão & o Dimensão 2 - Aplicabilidade do Subprocesso Organizacional & Média & 1 & 2 & 3 & 4 & 5 \\
\hline & $\begin{array}{l}\text { O processo permitiu aumentar o alinhamento estratégico (entre o planejamento } \\
\text { estratégico e os processos organizacionais) através do uso do Balanced Scorecard e } \\
10 \text { seus artefatos/ferramentas como proposto. }\end{array}$ & 4,43 & 0 & 0 & 0 & 4 & 3 \\
\hline & $\begin{array}{l}\text { O processo contribuiu para a manutenção de dados históricos das medições e } \\
1 \mathbf{1} \text { indicadores instanciados visando uma maior previsibilidade }\end{array}$ & 4,43 & 0 & 0 & 1 & 2 & 4 \\
\hline & $\begin{array}{l}\text { O processo facilitou o entendimento a cerca das questões de coleta, publicação e } \\
12 \text { análise de resultados das medições e indicadores instanciados. }\end{array}$ & 5,00 & 0 & 0 & 0 & 0 & 7 \\
\hline & $\begin{array}{l}\text { O processo tornou claras as responsabilidades dos envolvidos na rotina do } \\
13 \text { gerenciamento quantitativo. }\end{array}$ & 4,29 & 0 & 0 & 1 & 3 & 3 \\
\hline & $\begin{array}{l}\text { O processo reduziu o esforço gasto em atividades de coleta de informações das } \\
14 \text { medições e indicadores instanciados. }\end{array}$ & 4,71 & 0 & 0 & 0 & 2 & 5 \\
\hline & $\begin{array}{l}\text { O processo reduziu a duplicidade de informações das medições e indicadores } \\
15 \text { instanciados. }\end{array}$ & 4,57 & 0 & 0 & 1 & 1 & 5 \\
\hline 16 & 16 O processo aumentou a acuracidade das medições e indicadores instanciados. & 4,29 & 0 & 0 & 2 & 1 & 4 \\
\hline
\end{tabular}

De uma forma geral, esta dimensão do questionário obteve resultados satisfatórios. Já que, a maioria das respostas em todas as questões tende para "Concordo" e "Concordo Totalmente". 
Resultado satisfatório foi obtido na questão 10 visto que o mesmo corrobora com a visão de autores como Vasques (Vasques, 2006) e Goethert (Goethert, 2003) que afirmam existir um maior alinhamento estratégico quando se utiliza o BSC para derivação de medições e indicadores de desempenho.

Houve unanimidade na questão 12, ao afirmarem que o processo proposto facilita o entendimento sobre coleta, publicação e análise dos indicadores e medições instanciados, fato associado à utilização do processo com uma ferramenta de apoio específica para automatizar coleta, análise e publicação de medições e indicadores de desempenho.

A terceira dimensão do questionário visava identificar a aplicabilidade do Subprocesso Análise de Resultados. A consolidação dos resultados se encontra na Tabela 3.

Tabela 3. Resultados Questionário - Dimensão 3

\begin{tabular}{|c|c|c|c|c|c|c|c|}
\hline Questão & Dimensão 3 - Aplicabilidade do Subprocesso Análise de Resultados & Média & 1 & 2 & 3 & 4 & 5 \\
\hline & $\begin{array}{l}\text { O processo de Análise de Resultados proporcionou um correto entendimento dos } \\
7 \text { problemas para a tomada de decisões. }\end{array}$ & 4,75 & 0 & 0 & 0 & 4 & 3 \\
\hline & $\begin{array}{l}\text { Os resultados dos indicadores produzidos segundo o processo auxiliaram na tomada de } \\
8 \text { ações corretivas. }\end{array}$ & 4,50 & 0 & 0 & 1 & 2 & 4 \\
\hline & $\begin{array}{l}\text { A visibilidade compartimentada dos resultados propiciou uma análise individual e } \\
9 \text { imparcial. }\end{array}$ & 4,25 & 0 & 0 & 2 & 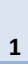 & 4 \\
\hline
\end{tabular}

As três questões desta dimensão do questionário apresentam resultados satisfatórios. Apesar da não obtenção de unanimidade em nenhuma das questões desta dimensão, existe uma forte tendência à concordância com as questões.

De acordo com a percepção dos respondentes, houve correto entendimento acerca dos problemas para a tomada de decisões como pode ser observado pelo resultado da questão 17. Nas questões 18 e 19 foram obtidas algumas respostas no meio da escala, isto abre a possibilidade de considerar que seus respondentes não sabem opinar sobre o tema.

O processo de tomada de decisão e análise de resultados necessita de um ciclo de vida maior para que possa ser devidamente avaliado. Tendo em vista as restrições de prazo para conclusão desta pesquisa, não foi possível acompanhar as análises e tomada de decisões em longo prazo. Ainda assim, na percepção dos respondentes, os resultados são satisfatórios visto que as médias estão entre a faixa de "Concordo" e "Concordo totalmente”.

Já a quarta dimensão do questionário visava identificar a aplicabilidade do Subprocesso Projetos e Sistemas, cuja consolidação dos resultados se encontra na Tabela 4.

Tabela 4. Resultados Questionário - Dimensão 4

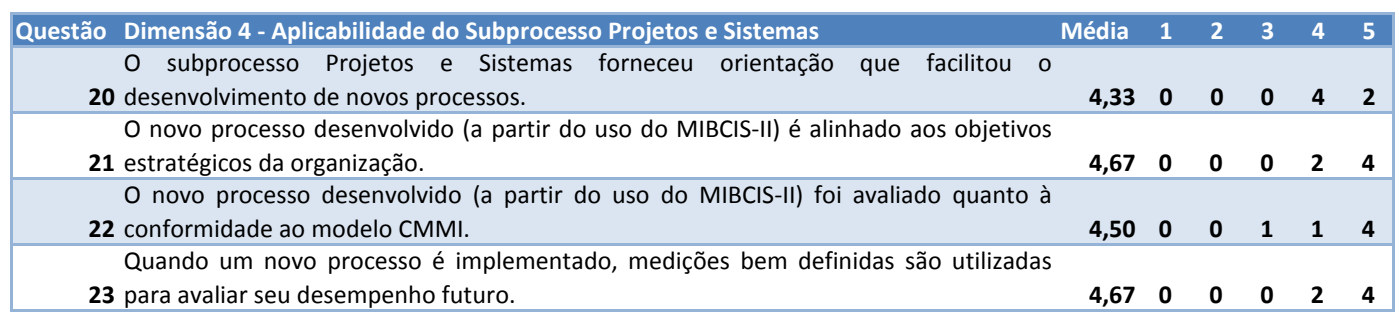


A avaliação do Subprocesso Projetos e Sistemas, responsável pela criação de processos, obteve resultados satisfatórios como pode ser visto na Tabela 4.

Na questão 20, um bom resultado foi obtido, visto que segundo a percepção dos respondentes, o desenvolvimento de novos processos foi facilitado seguindo as atividades do subprocesso Projetos e Sistemas. Desempenho satisfatório também foi observado na questão 21 indicando que os processos desenvolvidos possuem alinhamento estratégico, corroborando com Vasques (Vasques, 2006), que afirma que a integração entre BSC, CMMI e Six Sigma provê alinhamento estratégico para programas de melhoria.

A questão 22 obteve resultado satisfatório. O processo proposto possui uma atividade específica para avaliação de conformidade com o modelo CMMI que conta com o apoio de um artefato específico para facilitar esta avaliação. Esta atividade não estava presente no MIBCIS (Covatti, 2007) e se mostrou eficaz, segundo a percepção dos respondentes, no processo proposto.

A questão 23 também obteve resultados satisfatórios no que diz respeito à definição de medições para avaliação do desempenho dos processos. Essas medições são essenciais, pois são a base para a melhoria contínua nos processos.

Os resultados obtidos para avaliação do Subprocesso de Melhoria Contínua, quinta dimensão do questionário, são expostos na Tabela 5.

Tabela 5. Resultados Questionário - Dimensão 5

\begin{tabular}{|c|c|c|c|c|c|c|c|}
\hline Questão & Dimensão 5 - Aplicabilidade do Subprocesso de Melhoria Contínua & Média & 1 & 2 & 3 & 4 & 5 \\
\hline & $\begin{array}{l}\text { O subprocesso de Melhoria Contínua facilita a implementação de melhorias no } \\
24 \text { framework de processos da organização. }\end{array}$ & 4,33 & 0 & 0 & 1 & 2 & 3 \\
\hline & $\begin{array}{l}\text { As melhorias implementadas segundo o subprocesso de Melhoria Contínua } \\
25 \text { melhoraram o desempenho do processo alvo de melhoria. }\end{array}$ & 4,83 & 0 & 0 & 0 & 1 & 5 \\
\hline & $\begin{array}{l}\text { As melhorias de processo implementadas mantém alinhamento estratégico e } \\
26 \text { aderência ao modelo CMMI. }\end{array}$ & 4,67 & 0 & 0 & 0 & 2 & 4 \\
\hline
\end{tabular}

As questões desta dimensão do questionário são similares às questões da dimensão anterior, Subprocesso Projetos e Sistemas, e os resultados demonstram coerência dos respondentes.

Resultado satisfatório foi obtido na questão 24, indicando que as atividades do processo para implementação de melhorias são eficazes assim como na questão 20 da dimensão 4.

Bom resultado também foi obtido na questão 26, indicando que as melhorias de processo mantém alinhamento estratégico e aderência ao modelo CMMI, este resultado é coerente com as questões 21 e 22 da dimensão 4.

Por fim, a última dimensão do questionário visava identificar, de uma forma geral, aspectos relevantes do trabalho como um todo. Os resultados das questões desta dimensão se encontram na Tabela 6.

Tabela 6. Resultados Questionário - Dimensão 6

\begin{tabular}{|c|c|c|c|c|c|c|}
\hline Questão Dimensão 6 - Avaliação Geral do Processo & Média & 1 & 2 & 3 & 4 & 5 \\
\hline 29 O processo MIBCIS-II atende os objetivos a que se propõe. & 4,80 & 0 & 0 & 0 & 2 & 8 \\
\hline 30 A relação entre esforço de execução do processo e resultados obtidos é satisfatória. & 4,60 & 0 & 0 & 0 & 4 & 6 \\
\hline 31 O processo MIBCIS-II se mostra útil para a organização. & 4,80 & 0 & 0 & 0 & 2 & 8 \\
\hline
\end{tabular}


Resultados satisfatórios foram obtidos nas questões 29 e 31, com uma média elevada, tendendo à resposta "Concordo Totalmente”. Na questão 29, pode-se perceber que, segundo a percepção dos respondentes, o processo atende aos objetivos propostos.

Na questão 31, avaliam que o processo se mostra útil para a organização. Estes resultados são positivos visto que o objetivo principal do estudo de caso realizado é avaliar a aplicabilidade do processo em uma organização.

Um bom resultado também foi obtido na questão 30, porém com uma média ligeiramente inferior. Segundo a percepção dos respondentes pode-se perceber que o processo produz resultados satisfatórios que compensam o esforço de sua execução.

\section{Considerações finais}

Qualidade no desenvolvimento de sistemas de informação é uma peça fundamental para o funcionamento das organizações modernas. A partir da qualidade do processo é possível reduzir custos e oferecer produtos de também melhor qualidade. Custo e qualidade são diferenciais muito importantes no mercado globalizado de ampla concorrência.

Os temas abordados neste artigo são, portanto, considerados de relevância significativa para a pesquisa em qualidade de software e vem despertando crescente interesse tanto no meio acadêmico quanto na indústria de software. Esta pesquisa buscou evoluir a integração entre BSC, CMMI e Six Sigma, tendo como referência básica o MIBCIS (Covatti, 2007), de forma a construir um processo instanciável para as organizações de desenvolvimento de software.

A proposta de um processo integrado de gerenciamento quantitativo para melhoria de processos visa contribuir para área de engenharia de software ao preencher uma lacuna existente entre a integração dos métodos e modelos propostos utilizando uma ferramenta de especificação própria para processos de software. Esta necessidade é corroborada por Covatti (Covatti, 2007), Siviy (Siviy, 2005) e Vasques (Vasques, 2006).

Além disso, este estudo apresenta novos dados empíricos e busca também contribuir para a pesquisa na área de qualidade de software através do emprego de métodos qualitativos de pesquisa científica. Pode-se justificar o uso de métodos qualitativos, tais como o estudo de caso, aqui empregado, pelo fato de esta pesquisa envolver um estudo para aplicação do processo proposto no seu contexto real, com a descrição e a compreensão do estado da arte. Os resultados da aplicação do processo em uma organização de software foram considerados satisfatórios e o objetivo do estudo de caso foi atingido. Oportunidades de melhoria no processo foram identificadas e em sua maioria dizem respeito ao processo de análise de medições onde a exploração de técnicas estatísticas se faz necessária para alcançar melhores resultados.

\section{Referências}

Ahern, Dennis; Clouse, Aaron; Turner, Richard. CMMI® Distilled: A Pratical Introduction to Integrated Process Improvement, Segunda edição. Addison Wesley, 2003, 336pp. 
Covatti, A. MIBCIS - Método de Integração entre BSC, CMMI e Six Sigma utilizando GQM no suporte à definição de métricas. Programa de Pós-graduação em Ciência da Computação, PUC-RS 2007

Donegan, Paula et. all. Métricas de Software: Um Mapeamento entre Six Sigma e CMMI. SIMPROS2005

Eckes, George. A Revolução 6 Sigma. Rio de Janeiro: Editora Campus, 2001.

Goethert, Wolfhart; Fisher Matt. Deriving Enterprise-Based Measures Using the Balance Scorecard and Goal-Driven Measurement Techniques. Institute Carnegie Mellon University. October 2003.

Hayes Bruce. Introduction to Six Sigma for Software ... The Third Wave. Institute Carnegie Mellon University. Disponível em http://www.sei.cmu.edu/sema/pdf/sdc/hayes.pdf Acessado em junho de 2007.

Kaplan, R.; Norton, D. A estratégia em ação: balanced scorecard. Rio de Janeiro: Elsevier, 1997.

Oliveira, Sandro Ronaldo Bezerra et. all. Uma Proposta de Um Ambiente de Implementação de Processo de Software. INFOCOMP. Journal of Computer Science V4.N1 Disponível em http://www.dcc.ufla.br/infocomp/artigos/v4.1/art09.pdf. Acessado em dezembro de 2007.

Pickerill Jay. Implementing the CMMI in a Six Sigma World.Institute Carnegie Mellon University. Disponível em: http://www.sei.cmu.edu/cmmi/presentations/sepg05.presentations/pickerill.pdf. Acesso em setembro de 2008.

Siviy, Jeannine M; Penn, M. Lynn; Harper, Erin. Relationships between CMMI and Six Sigma. Institute Carnegie Mellon University. Disponível em: http://www.sei.cmu.edu/publications/documents/05.reports/05tn005.html. Acesso em Setembro de 2008.

Fehlmann, Thomas M. Six Sigma for Software. iSixSigma. Disponível em http://www.isixsigma.com/itra/ . Acessado em junho de 2007.

Vasques, Renato. BSC, CMMI e Six Sigma como construir altos níveis de maturidade e desempenho de forma integrada. MundoPM. Número 09. Jun/Jul 2006. 